\title{
Ansiedade em adolescentes no contexto da pandemia por COVID-19
}

\author{
Anxiety in adolescents in the context of the pandemic by COVID-19 \\ Ansiedad en adolescentes en el contexto de la pandemia por COVID-19
}

Recebido: 09/05/2021 | Revisado: 12/05/2021 | Aceito: 17/05/2021 | Publicado: 19/05/2021

\author{
Kahena de Oliveira Rego \\ ORCID: https://orcid.org/0000-0001-5157-1047 \\ Universidade Tiradentes, Brasil \\ E-mail: kahena.oliveira.rego@gmail.com. \\ Juliana Leal Freitas Maia \\ ORCID: https://orcid.org/0000-0002-1751-4572 \\ Universidade Tiradentes, Brasil \\ E-mail: juliana.maia@souunit.com.br
}

\section{Resumo}

O objetivo do presente estudo foi identificar a literatura existente relacionada à ansiedade em adolescentes no contexto da pandemia por COVID-19. Como metodologia utilizada para escrever esse artigo foi a revisão sistemática da literatura com material pesquisado que estivesse disponível nas bases de dados MEDLINE e Pubmed disponíveis de forma completa gratuitamente publicados a partir de 2020. Os resultados foram compostos com discussão de 16 artigos que foram selecionados após leitura e análise minuciosa do conteúdo. Para uma melhor organização da discussão de resultados, os dados foram divididos em três tópicos específicos, com agrupamento dos mesmos conforme a temática relativa às informações, como segue: 1) Prevenção da ansiedade em adolescente frente à pandemia por COVID-19; 2) Características da ansiedade em adolescente frente à pandemia por COVID-19; 3) Estratégias inovadoras nas intervenções médicas em adolescente com ansiedade frente a pandemia por COVID-19. Conclui-se que a ansiedade foi muito prevalente entre adolescentes durante o surto de COVID-19, uma vez que tal pandemia gerou efeito multiplicador em diversas características da ansiedade. É importante que as autoridades de saúde identifiquem os grupos de alto risco de adoecimento psíquico, para implementar intervenções psiquiátricas e psicológicas adequadas. Ainda há pouca literatura que tenha foco na ansiedade em adolescentes durante a pandemia. Faz-se necessário, portanto, que surjam novos estudos com foco nas estratégias de redução de ansiedade associada a este contexto mundial, a fim de que seja minimizado o impacto negativo na qualidade de vida dos adolescentes.

Palavras-chave: Ansiedade; Adolescente; Psiquiatria; Infecções por Coronavirus.

\begin{abstract}
The aim of the present study was to identify the existing literature related to anxiety in adolescents in the context of the COVID19 pandemic. As a methodology used to write this article was the systematic review of the literature with researched material that was available in the MEDLINE and Pubmed databases, available in a completely free form, published from 2020. The results were composed with a discussion of 16 articles that were selected after thorough reading and analysis of the content. For a better organization of the discussion of results, the data were divided into three specific topics, with grouping them according to the theme related to the information, as follows: 1) Prevention of anxiety in adolescents facing the pandemic by COVID-19; 2) Characteristics of anxiety in adolescents facing the pandemic by COVID-19; 3) Innovative strategies in medical interventions in adolescents with anxiety in the face of the COVID-19 pandemic. It is concluded that anxiety was very prevalent among adolescents during the outbreak of COVID-19, since such a pandemic generated a multiplying effect on several characteristics of anxiety. It is important for health authorities to identify groups at high risk for mental illness in order to implement appropriate psychiatric and psychological interventions. There is still little literature that focuses on anxiety in adolescents during the pandemic. It is necessary, therefore, that new studies with a focus on anxiety reduction strategies associated with this world context emerge, in order to minimize the negative impact on adolescents' quality of life.
\end{abstract}

Keywords: Anxiety; Adolescent; Psychiatry; Coronavirus Infections.

\section{Resumen}

El objetivo del presente estudio fue identificar la literatura existente relacionada con la ansiedad en adolescentes en el contexto de la pandemia del COVID-19. Como metodología utilizada para la redacción de este artículo fue la revisión sistemática de la literatura con material investigado que estaba disponible en las bases de datos MEDLINE y Pubmed, disponible en forma completamente gratuita, publicado a partir de 2020. Los resultados se compusieron con una discusión de 16 artículos que fueron seleccionados después de una lectura y un análisis exhaustivos del contenido. Para una mejor organización de la discusión de resultados, los datos se dividieron en tres temas específicos, agrupándolos según el tema relacionado con la información, de la siguiente manera: 1) Prevención de la ansiedad en adolescentes frente a la pandemia por COVID-19; 2) Características de la ansiedad en adolescentes ante la pandemia COVID-19; 3) Estrategias innovadoras en intervenciones médicas en adolescentes 
Research, Society and Development, v. 10, n. 6, e39010615930, 2021

(CC BY 4.0) | ISSN 2525-3409 | DOI: http://dx.doi.org/10.33448/rsd-v10i6.15930

con ansiedad ante la pandemia COVID-19. Se concluye que la ansiedad fue muy prevalente entre los adolescentes durante el brote de COVID-19, ya que dicha pandemia generó un efecto multiplicador sobre varias características de la ansiedad. Es importante que las autoridades sanitarias identifiquen los grupos de alto riesgo de enfermedad mental para poder implementar las intervenciones psiquiátricas y psicológicas adecuadas. Todavía hay poca literatura que se centre en la ansiedad en los adolescentes durante la pandemia. Es necesario, por tanto, que surjan nuevos estudios con enfoque en estrategias de reducción de la ansiedad asociadas a este contexto mundial, con el fin de minimizar el impacto negativo en la calidad de vida de los adolescentes.

Palabras clave: Ansiedad; Adolescencia; Psiquiatría; Infecciones por Coronavirus.

\section{Introdução}

A ansiedade é conhecida como sendo uma condição emocional com componentes fisiológicos e psicológicos, integrando as experiências humanas no cotidiano e ainda alavancando o desempenho e ela passa a ser patológica quando é desproporcional à situação que a desencadeia, ou quando não existe um objeto específico ao qual se direciona. De acordo com a Organização Mundial da Saúde, existem cerca de 264 milhões de pessoas globalmente que sofrem de transtornos de ansiedade, o que representa um aumento de $15 \%$ desde 2005 (WHO, 2017).

Os transtornos de ansiedade são os transtornos psiquiátricos mais prevalentes (com uma prevalência mundial atual de 7,3\%) (Thibaut, 2017). Entre eles, as fobias específicas são as mais comuns, com uma prevalência de 10,3\%, sendo o transtorno do pânico (com ou sem agorafobia) o segundo mais comum com uma prevalência de 6,0\%, seguido pela fobia social (2,7\%) e transtorno de ansiedade generalizada (2,2\%). Torna-se importante salientar que, de modo geral, as mulheres são mais propensas a desenvolver distúrbios emocionais com início na adolescência; eles têm 1,5 a 2 vezes mais probabilidade do que os homens de ter um transtorno de ansiedade (Thibaut, 2017).

A pandemia causada pelo coronavírus (COVID-19) provocou impactos relevantes na vida de pessoas em todo o mundo, incluindo crianças e adolescentes, de uma forma sem precedentes (Gunnell, 2020). Em todo o planeta, uma atitude essencial para prevenção da infecção por COVID-19 tem sido o isolamento (Sher, 2020), e as estratégias de distanciamento social para proteger do risco de infecção (Singh, 2020). Desde janeiro de 2020, vários países começaram a implementar medidas de contenção ou bloqueio regionais e nacionais e uma das principais medidas tomadas foi o fechamento de escolas, institutos de ensino, sendo que essas circunstâncias inexoráveis, que estão além da experiência normal, podem levar ao estresse, à ansiedade e a um sentimento de desamparo em todos (Singh, 2020).

Os transtornos de saúde mental, como a ansiedade, são responsáveis por 16\% da carga global de doenças e lesões entre as pessoas de 10 a 19 anos. Estima-se que 10-20\% das crianças e adolescentes em todo o mundo sofrem de problemas de saúde mental como a ansiedade. Globalmente, a ansiedade é a nona causa principal de doença e incapacidade em adolescentes de 15 a 19 anos e a sexta entre aqueles de 10 a 14 anos (Zhou, 2020). Recentemente, um estudo avaliou 1.036 crianças e adolescentes em quarentena na China na faixa etária de 6 a 15 anos, dos quais 112, 196 e 68 apresentavam depressão, ansiedade e ambos, respectivamente (Chen, 2020).

Pensando nisso, adolescentes podem estar altamente expostos a estressores psicossociais gerados pela pandemia e, uma vez que medidas de contenção da população para reduzir a disseminação do vírus sejam necessárias, eles podem ser potencialmente afetados pela interrupção na rotina da vida diária. Sem intervenções psicológicas adequadas, a ansiedade entre os adolescentes muitas vezes persiste na idade adulta e aumentam os fatores de risco de doenças relacionadas à idade (Figueiredo, 2020).

Nesse contexto, em comparação com adultos, a pandemia pode vir a ter consequências adversas aumentadas a longo prazo em adolescentes, que são um grupo vulnerável e podem ter consequências múltiplas na vida: estresse crônico e agudo, 
Research, Society and Development, v. 10, n. 6, e39010615930, 2021

(CC BY 4.0) | ISSN 2525-3409 | DOI: http://dx.doi.org/10.33448/rsd-v10i6.15930

preocupação com suas famílias, luto inesperado, interrupção repentina da escola e confinamento em casa em muitos países, maior tempo de acesso à internet, além de preocupação com o futuro econômico de sua família e país (Guessoum, 2020).

O toque social é essencial para desenvolver cognição, emoções, apego e relacionamentos (GUESSOUM, 2020). Além disso, também contribui para a regulação fisiológica das respostas do corpo aos estressores agudos, bem como a outros desafios de curto prazo. Para Loades (2020), o distanciamento social e o fechamento de escolas podem resultar em um aumento da solidão em adolescentes cujos contatos sociais usuais são restringidos pelas medidas de contenção de doenças. Embora o isolamento social não seja necessariamente sinônimo de solidão, as primeiras pesquisas no contexto COVID-19 indicam que mais de um terço dos adolescentes relatam altos níveis de solidão deixando evidentes as ligações já bem estabelecidas entre solidão e saúde mental, podendo provocar elevação nos níveis de ansiedade (Loades, 2020).

Assim, o objetivo do presente estudo é identificar a literatura científica existente relacionada a ansiedade em adolescentes no contexto da pandemia por covid-19.

\section{Metodologia}

A metodologia utilizada para escrever esse artigo foi a revisão sistemática da literatura (Castro, 2001). A questão norteadora desta revisão foi: qual a literatura existente relacionada à ansiedade em adolescentes no contexto da pandemia por covid-19? Foi realizada uma busca minuciosa do material utilizado em periódicos das áreas correspondentes ao tema principal do artigo. Utilizou-se o material pesquisado que estivesse disponível nas seguintes bases de dados: Medical LiteratureAnalysisandRetrieval System Online (MEDLINE) e Pubmed que é um serviço da U. S. National Library of Medicine (NLM) (Galvão, 2014).

Foram estabelecidos como critérios de inclusão: artigos científicos completos gratuitamente disponíveis para consulta publicados a partir de 2020 (ano em que foi decretada a pandemia por covid-19 mundialmente); em língua inglesa; possuir relação direta com o objetivo proposto, estando publicados em periódicos de relevância científica comprovada por fator de impacto. Sendo que os critérios de exclusão foram artigos disponibilizados somente em fotocópia; teses, dissertações e monografias; e os que se encontravam repetidos em bases de dados diferentes.

A pesquisa dos artigos utilizados foi feita de março a abril de 2021, sendo eleitos os seguintes descritores conforme MESH da PUBMED: Anxiety, Adolescent, Psychiatry e Coronavirus Infections.

Nesta revisão sistemática foram encontrados 7 artigos na base de dados MEDLINE, enquanto na base de dados Pubmed foram encontrados 122 artigos. Diante do número de artigos, que totalizou 129, foram excluídos artigos disponibilizados somente em fotocópia; teses, dissertações e monografias e os que se encontravam repetidos em bases de dados diferentes, permanecendo 37 estudos. Desses, foram selecionados os artigos científicos publicados a partir de 2020, em língua inglesa; disponíveis para consulta gratuita; publicados em periódicos de relevância científica comprovada por fator de impacto, possuindo relação direta com o objetivo proposto, permanecendo 2 artigos na base de dados MEDLINE e 14 artigos na base de dados Pubmed, num total de 16 artigos.

Os textos foram selecionados com base em resultados que guardavam relação com a questão norteadora, reorganizados e sintetizados para melhor compreensão e para a construção dos argumentos da discussão.

\section{Resultados}

Após uma revisão sistematizada dos dados por meio da leitura minuciosa dos artigos, além da aplicação dos critérios de inclusão e exclusão descritos na metodologia foram selecionados aqueles artigos contidos na MEDLINE e na Pubmed que pudessem guardar relação com o objetivo do estudo, respondendo a questão norteadora desta pesquisa. 
Os 16 textos foram então submetidos a uma tabulação em formato de quadro analítico onde os dados foram sintetizados e dispostos organizadamente de forma sistemática, para melhor exposição das informações:

Quadro 1 - Desmembramento das informações dos artigos selecionados.

\begin{tabular}{|c|c|c|c|c|}
\hline Título & $\begin{array}{c}\text { Periód. / } \\
\text { ano }\end{array}$ & Tipo / metodo & Objetivos & Conclusões \\
\hline $\begin{array}{l}\text { Impact COVID-19 and } \\
\text { lockdownon mental } \\
\text { health of adolescents: } \\
\text { Narrative review } \\
\text { recommendations. }\end{array}$ & $\begin{array}{l}\text { Psychiatry } \\
\text { research / } \\
2020 \\
\text { MEDLIN } \\
\text { E }\end{array}$ & $\begin{array}{l}\text { Revisão } \\
\text { narrativa }\end{array}$ & $\begin{array}{l}\text { Revisar narrativamente } \\
\text { artigos sobre a saúde } \\
\text { mental de adolescentes } \\
\text { afetados pela pandemia de } \\
\text { COVID-19. }\end{array}$ & $\begin{array}{l}\text { Estabelecer políticas } \\
\text { inovadoras de saúde mental } \\
\text { para adolescentes, com redes } \\
\text { colaborativas diretas e digitais } \\
\text { de voluntários. }\end{array}$ \\
\hline $\begin{array}{l}\text { COVID-19 Impacts on } \\
\text { Child and Youth Anxiety } \\
\text { and Depression: } \\
\text { Challenges } \\
\text { Opportunities }\end{array}$ & $\begin{array}{l}\text { Revuecana } \\
\text { dienne de } \\
\text { psychiatria } \\
\text { / } 2020 \text { / } \\
\text { MEDLIN } \\
\text { E }\end{array}$ & $\begin{array}{l}\text { Revisão } \\
\text { narrativa }\end{array}$ & $\begin{array}{l}\text { Relatar os desafios e } \\
\text { oportunidades dos } \\
\text { Impactos do COVID-19 na } \\
\text { ansiedade e depressão de } \\
\text { crianças e jovens. }\end{array}$ & $\begin{array}{l}\text { Atentar à saúde mental de } \\
\text { adolescentes com depressão e } \\
\text { ansiedade preexis-tentes; além } \\
\text { de intervenções para prevenir } \\
\text { os efeitos. }\end{array}$ \\
\hline $\begin{array}{l}\text { Adolescent psychiatric } \\
\text { disorders during the } \\
\text { COVID-19 pandemic and } \\
\text { lockdown. }\end{array}$ & $\begin{array}{l}\text { Psychiatry } \\
\text { research / } \\
2020\end{array}$ & $\begin{array}{l}\text { Revisão de } \\
\text { literatura }\end{array}$ & $\begin{array}{l}\text { Revisar a literatura sobre } \\
\text { transtornos psiquiátricos } \\
\text { em adolescentes relacio- } \\
\text { nados à pandemia e ao } \\
\text { bloqueio. }\end{array}$ & $\begin{array}{l}\text { Vulnerabilidade familiar, social } \\
\text { e as habilidades de } \\
\text { enfrentamento do adoles-cente, } \\
\text { são relacionados a sua saúde } \\
\text { mental na crise. }\end{array}$ \\
\hline $\begin{array}{l}\text { Rapid Systematic Re- } \\
\text { view: The Impact of } \\
\text { Social Isolation and } \\
\text { Loneliness on the Mental } \\
\text { Health of Children and } \\
\text { Adolescents in Context } \\
\text { COVID-19. }\end{array}$ & $\begin{array}{l}\text { Journal } \\
\text { American } \\
\text { Academy } \\
\text { Childand } \\
\text { Adolescen } \\
\text { tPsychiatr } \\
\text { y / } 2020\end{array}$ & $\begin{array}{l}\text { Revisão } \\
\text { Sistemática } \\
\text { Rápida }\end{array}$ & $\begin{array}{l}\text { Estabelecer o que se sabe } \\
\text { sobre como as medidas de } \\
\text { solidão e contenção de } \\
\text { doenças impactam na saúde } \\
\text { mental de crianças e } \\
\text { adolescentes. }\end{array}$ & $\begin{array}{l}\text { Serviços clínicos devem } \\
\text { oferecer apoio preventivo e } \\
\text { intervenção precoce sempre } \\
\text { que possível e estar preparados } \\
\text { para o aumento dos problemas } \\
\text { de saúde mental. }\end{array}$ \\
\hline $\begin{array}{l}\text { Prevalence and socio- } \\
\text { demographic correlates of } \\
\text { psychological health } \\
\text { problems in Chinese } \\
\text { adolescentes during the } \\
\text { outbreak of COVID-19. }\end{array}$ & $\begin{array}{l}\text { Europeanc } \\
\text { hild\&adol } \\
\text { escentpsyc } \\
\text { hiatry / } \\
2020\end{array}$ & $\begin{array}{l}\text { Estudo } \\
\text { transversal } \\
\text { quantitativo }\end{array}$ & $\begin{array}{l}\text { Avaliar a taxa de } \\
\text { prevalência e correlatos } \\
\text { sociodemográficos de } \\
\text { sintomas depressivos e de } \\
\text { ansiedade } \\
\text { adolescentes entre } \\
\text { afetados pelo surto de } \\
\text { COVID-19. }\end{array}$ & $\begin{array}{l}\text { Alta prevalência de problemas } \\
\text { psicológicos } \\
\text { adolescentes, podendo estar } \\
\text { associados ao COVID-19, } \\
\text { sugerindo que o governo } \\
\text { precisa dar mais atenção no } \\
\text { combate o COVID-19. }\end{array}$ \\
\hline
\end{tabular}




\begin{tabular}{|c|c|c|c|c|}
\hline $\begin{array}{l}\text { COVID-19 pandemic } \\
\text { impact on children } \\
\text { andadolescents' mental } \\
\text { health: Biological, } \\
\text { environmental, and social } \\
\text { factors. }\end{array}$ & $\begin{array}{l}\text { Progress in } \\
\text { neuropsyc } \\
\text { hopharmac } \\
\text { ologybio- } \\
\text { logicalpsy } \\
\text { chiatry / } \\
2021\end{array}$ & $\begin{array}{l}\text { Revisão } \\
\text { literatura }\end{array}$ & $\begin{array}{l}\text { Discutir e alertar os agentes } \\
\text { de saúde pública e } \\
\text { governamentais sobre a } \\
\text { necessidade de vigilância } \\
\text { aos adolescentes. }\end{array}$ & $\begin{array}{l}\text { Acompanhamento } \\
\text { adolescentes, família } \\
\text { cuidadores, para criar } \\
\text { estratégias que minimizem os } \\
\text { efeitos da pandemia do } \\
\text { COVID-19. }\end{array}$ \\
\hline $\begin{array}{l}\text { Aninvestigation of mental } \\
\text { health status of children } \\
\text { andadolescents in China } \\
\text { during the outbreak of } \\
\text { COVID-19. }\end{array}$ & $\begin{array}{l}\text { Journalofa } \\
\text { ffectivedis } \\
\text { orders / } \\
2020\end{array}$ & $\begin{array}{l}\text { Estudo } \\
\text { transversal } \\
\text { quantitativo }\end{array}$ & $\begin{array}{l}\text { Demonstrar os efeitos } \\
\text { psicológicos do COVID-19 } \\
\text { em crianças e adolescentes } \\
\text { associados à pandemia. }\end{array}$ & $\begin{array}{l}\text { Abordar o sofrimento } \\
\text { emocional de adolescentes } \\
\text { durante a pandemia, e formular } \\
\text { intervenções direcionadas com } \\
\text { base nos fatores de influência. }\end{array}$ \\
\hline $\begin{array}{l}\text { Mental health and its } \\
\text { correlates among children } \\
\text { andadolescents during } \\
\text { COVID-19 school } \\
\text { closure: The importance } \\
\text { of parentechilddis } \\
\text { cussion." }\end{array}$ & $\begin{array}{l}\text { Journalofa } \\
\text { ffectivedis } \\
\text { orders / } \\
2021\end{array}$ & $\begin{array}{l}\text { Estudo } \\
\text { transversal } \\
\text { quantitativo }\end{array}$ & $\begin{array}{l}\text { Investigar rigorosamente } \\
\text { sobre a saúde mental dos } \\
\text { alunos durante a pandemia } \\
\text { do Coronavírus. }\end{array}$ & $\begin{array}{l}\text { A comunicação aberta entre } \\
\text { pais e filhos sobre a pandemia } \\
\text { deve ser encorajada para ajudar } \\
\text { adolescentes a lidar com } \\
\text { problemas de saúde mental. }\end{array}$ \\
\hline $\begin{array}{l}\text { Risk, resilience, } \\
\text { psychological distress, } \\
\text { and anxiety at the } \\
\text { beginning of the COVID- } \\
19 \text { pandemic in Germany. }\end{array}$ & $\begin{array}{l}\text { BrainBeha } \\
\text { v / } 2020\end{array}$ & $\begin{array}{l}\text { Estudo } \\
\text { transversal } \\
\text { quantitativo } \\
\text { (pesquisa } \\
\text { online) }\end{array}$ & $\begin{array}{l}\text { Rastrear a população da } \\
\text { Alemanha quanto ao } \\
\text { impacto sobre a saúde } \\
\text { mental na pandemia de } \\
\text { COVID-19 e analisar } \\
\text { possíveis fatores de risco e } \\
\text { proteção. }\end{array}$ & $\begin{array}{l}\text { Manter um estilo de vida } \\
\text { saudável com contatos sociais, } \\
\text { e, informações sobre onde obter } \\
\text { tratamento médico, } \\
\text { necessário, parecem ajudar. }\end{array}$ \\
\hline $\begin{array}{l}\text { Generalized anxiety and } \\
\text { depressive symptoms in } \\
\text { various age groups during } \\
\text { the COVID-19 lockdown } \\
\text { in Poland. Specific } \\
\text { predictors and differences } \\
\text { in symptoms severity. }\end{array}$ & $\begin{array}{l}\text { ComprPsy } \\
\text { chiatry / } \\
2021\end{array}$ & $\begin{array}{l}\text { Estudo } \\
\text { transversal } \\
\text { quantitativo }\end{array}$ & $\begin{array}{l}\text { Investigar as diferenças nos } \\
\text { níveis de sintomas de } \\
\text { ansiedade depressiva e } \\
\text { generalizada em quatro } \\
\text { grupos de idade na } \\
\text { população polonesa durante } \\
\text { o bloqueio COVID-19. }\end{array}$ & $\begin{array}{l}\text { Os adolescentes com } \\
\text { dificuldades de relacionamento } \\
\text { entre os membros da família } \\
\text { são os mais vulneráveis aos } \\
\text { sintomas de ansiedade } \\
\text { generalizada durante o bloqueio } \\
\text { COVID-19. }\end{array}$ \\
\hline $\begin{array}{l}\text { Prevalence of Anxi-ety } \\
\text { and Associated Factors } \\
\text { for Chine-se Adolescents } \\
\text { during the COVID-19 } \\
\text { Outbreak. }\end{array}$ & $\begin{array}{l}\text { Psychiatry } \\
\text { ClinNeuro } \\
\text { sci / } 2020\end{array}$ & $\begin{array}{l}\text { Revisão } \\
\text { literatura }\end{array}$ & $\begin{array}{l}\text { Analisar adolescentes que } \\
\text { podem ter sido esquecidos } \\
\text { durante a pandemia causada } \\
\text { pelo coronavírus. }\end{array}$ & $\begin{array}{l}\text { A ansiedade foi prevalente } \\
\text { entre adolescentes durante o } \\
\text { surto de COVID-19. }\end{array}$ \\
\hline
\end{tabular}




\begin{tabular}{|c|c|c|c|c|}
\hline $\begin{array}{l}\text { Identifying the impact of } \\
\text { the confinement of Covid- } \\
19 \text { on emotional mood } \\
\text { and behavioural } \\
\text { dimensions in } \\
\text { childrenandadoles- } \\
\text { centswithattentiondeficith } \\
\text { yperactivitydisorder } \\
\text { (ADHD). }\end{array}$ & $\begin{array}{l}\text { Psychiatry } \\
\text { research / } \\
2021\end{array}$ & $\begin{array}{l}\text { Estudo } \\
\text { transversal } \\
\text { quantitativo } \\
\text { (pesquisa } \\
\text { online) }\end{array}$ & $\begin{array}{l}\text { Examinar o efeito do } \\
\text { bloqueio em adoles-centes } \\
\text { com TDAH com base nas } \\
\text { mudanças no grau de } \\
\text { gravidade de seu estado de } \\
\text { humor emocional e } \\
\text { compor-tamentos, durante } \\
\text { a pandemia coronavírus. }\end{array}$ & $\begin{array}{l}\text { A abordagem } \\
\text { proporcionou } \\
\text { individuação pontual dos } \\
\text { domínios afetados e os } \\
\text { benefícios indiretos produz- } \\
\text { zidos pela condição de restrição } \\
\text { em adolescentes com TDAH, } \\
\text { tendo em vista a da rotina } \\
\text { regular. }\end{array}$ \\
\hline $\begin{array}{l}\text { Debate: Recognising and } \\
\text { responding to the mental } \\
\text { health needs of Young } \\
\text { people in the era of } \\
\text { COVID-19. }\end{array}$ & $\begin{array}{l}\text { Childanda } \\
\text { dolescent } \\
\text { mental } \\
\text { health / } \\
2020\end{array}$ & $\begin{array}{l}\text { Revisão } \\
\text { narrativa }\end{array}$ & $\begin{array}{l}\text { Debater o tema Reco- } \\
\text { nhecendo e respondendo às } \\
\text { necessidades de saúde } \\
\text { mental dos jovens na era } \\
\text { COVID-19. }\end{array}$ & $\begin{array}{l}\text { Necessário entender se o } \\
\text { tratamento tele-psiquiatria é } \\
\text { eficaz contra os danos da } \\
\text { pandemia para promover a } \\
\text { saúde mental dos jovens. }\end{array}$ \\
\hline $\begin{array}{l}\text { Depression, anxiety and } \\
\text { associated fac-tors among } \\
\text { Chinese adolescentes } \\
\text { during COVID-19 out- } \\
\text { break: a comparison two } \\
\text { crosssectional st }\end{array}$ & $\begin{array}{l}\text { TranslPsyc } \\
\text { hiatry / } \\
2021\end{array}$ & $\begin{array}{l}\text { Estudo } \\
\text { transversal } \\
\text { quantitativo }\end{array}$ & $\begin{array}{l}\text { Examinar as mudanças na } \\
\text { ansiedade entre } \\
\text { adolescentes chineses } \\
\text { durante a epidemia de } \\
\text { COVID-19 e explorar os } \\
\text { fatores associados à } \\
\text { depressão e ansiedade. }\end{array}$ & $\begin{array}{l}\text { Triagem regular e intervenções } \\
\text { apropriadas são urgentes e } \\
\text { necessárias para reduzir o risco } \\
\text { de distúrbios emocionais entre } \\
\text { adolescentes durante e após os } \\
\text { surtos de COVID-19. }\end{array}$ \\
\hline $\begin{array}{l}\text { Debate: Student mental } \\
\text { health matters }- \text { the } \\
\text { heightened need for } \\
\text { school-based mental } \\
\text { health in the era of } \\
\text { COVID-19. }\end{array}$ & $\begin{array}{l}\text { Childanda } \\
\text { dolescent } \\
\text { mental } \\
\text { health / } \\
2020\end{array}$ & $\begin{array}{l}\text { Revisão } \\
\text { narrativa }\end{array}$ & $\begin{array}{l}\text { Debater questões de saúde } \\
\text { mental de alunos frente a } \\
\text { necessidade de saúde } \\
\text { mental baseada na escola na } \\
\text { era COVID-19. }\end{array}$ & $\begin{array}{l}\text { As escolas também precisam } \\
\text { apoiar os professores de forma } \\
\text { criativa durante este período } \\
\text { sem precedentes. }\end{array}$ \\
\hline $\begin{array}{l}\text { Symptomsofanxietydisor } \\
\text { ders in Iranian } \\
\text { adolescents with hearing } \\
\text { loss during the COVID- } \\
19 \text { pandemic. }\end{array}$ & $\begin{array}{l}\text { BMC } \\
\text { Psychiatry } \\
\text { / } 2021\end{array}$ & $\begin{array}{l}\text { Estudo } \\
\text { transversal } \\
\text { quantitativo }\end{array}$ & $\begin{array}{l}\text { Investigar a presença de } \\
\text { sintomas de transtornos de } \\
\text { ansiedade em adolescentes } \\
\text { com deficiência auditiva } \\
\text { durante a pandemia de } \\
\text { COVID-19. }\end{array}$ & $\begin{array}{l}\text { Presença de sintomas de } \\
\text { transtornos de ansiedade em } \\
\text { uma amostra de adolescentes, } \\
\text { especialmente em adolescentes } \\
\text { surdos, durante a pandemia de } \\
\text { COVID-19. }\end{array}$ \\
\hline
\end{tabular}

Fonte: Dados encontrados mediante pesquisa bibliográfica. Elaboração própria (2021).

Nesse contexto, pela intenção de singularizar os achados (de uma forma resumida) dos artigos selecionados, através da elaboração do quadro acima, tornou-se possível uma concentração dos dados para facilitar a construção analítica da discussão em torno da temática deste artigo de revisão sistemática. 
Research, Society and Development, v. 10, n. 6, e39010615930, 2021

(CC BY 4.0) | ISSN 2525-3409 | DOI: http://dx.doi.org/10.33448/rsd-v10i6.15930

\section{Discussão}

Para uma melhor organização da discussão acerca da análise dos artigos obtidos nos resultados, os dados foram divididos em três tópicos específicos, com agrupamento dos mesmos conforme a temática relativa às informações, como segue:

\section{Características da ansiedade em adolescente frente à pandemia por COVID-19}

Como um grupo especialmente sensível, os adolescentes são particularmente dignos de atenção, principalmente porque, diante da exposição a eventos estressantes, eles têm grande possibilidade de desenvolver sintomas de ansiedade e depressão (Qi, 2020)

Segundo a pesquisa de Qi (2020), a ansiedade foi muito prevalente entre adolescentes durante o surto de COVID-19, uma vez que tal pandemia gerou efeito multiplicador em diversas características da ansiedade. Nessa vertente, dadas as preocupações com relação ao contágio, a pandemia também pode estar associada à exacerbação de tipos específicos de ansiedade, incluindo fobias específicas, transtorno obsessivo-compulsivo e ansiedade generalizada relacionada a situações imprevisíveis e assustadoras; em contrapartida, o distanciamento social pode aliviar temporariamente alguns sintomas de ansiedade como a ansiedade social, ansiedade de desempenho relacionada aos trabalhos escolares ou agorafobia (Zhou, 2020).

Pensando ainda nos sintomas de ansiedade generalizada que tendem a emergir durante o distanciamento social provocado pelo COVID-19, os adolescentes com dificuldades de comunicação e relacionamento entre os membros da família são considerados como os mais vulneráveis (Ariapooran, 2021). Ariapooran (2021) constatou em seu estudo a presença de sintomas significativos de transtornos de ansiedade em uma amostra de adolescentes iranianos surdos durante a pandemia de COVID-19, e esses resultados podem ser relacionados com o processo de traumatização por privação de informação decorrente de informações inadequadas para a parcela da população composta de surdos (Ariapooran, 2021).

Tendo em vista essa perspectiva, a vulnerabilidade familiar, social, transtornos de estresse pós-traumático, depressão e ansiedade tem potencial de impacto desastroso na saúde mental de adolescentes, levando a necessidade de habilidades de enfrentamento individual (Guessoum, 2020). Ademais, pode-se inferir que a cobertura da imprensa / mídia também pode afetar adversamente a ansiedade e os sintomas depressivos, uma vez que informações falsas e relatórios falsos sobre COVID-19 podem agravar a ansiedade e os sintomas depressivos no público em geral (Guessoum, 2020).

\section{Prevenção da ansiedade em adolescente frente à pandemia por COVID-19}

A prevenção da ansiedade em adolescentes frente à pandemia por COVID-19 vem ganhando cada vez mais espaço na literatura. Psiquiatras, pediatras e provedores de cuidados primários precisam estar atentos à saúde mental da população de adolescentes, especialmente com ênfase aos sintomas de ansiedade nessa parcela da população e, nesse sentido, intervenções práticas e psicológicas bem estabelecidas podem ajudar a prevenir a ansiedade em adolescentes no contexto de isolamento social durante a pandemia de COVID-19 (Weisbrot, 2020).

Manter um estilo de vida saudável com contatos sociais e informações sobre onde obter tratamento médico, se necessário, parecem auxiliar os adolescentes a enfrentar melhor as situações e prevenir a ansiedade que possa surgir (Weisbrot, 2020). Courtney (2020) destaca a preponderância em atentar à saúde mental de adolescentes com depressão e ansiedade preexistentes; além de otimizar a intervenção para prevenir os efeitos negativos. Nessa perspectiva, a realização de triagens regulares e intervenções apropriadas são urgentes e necessárias para reduzir o risco de distúrbios emocionais entre adolescentes durante e após os surtos iniciais de COVID-19 (Chen, 2021), onde as escolas também precisam apoiar os professores de forma criativa durante este período sem precedentes (Weisbrot, 2020). 
Research, Society and Development, v. 10, n. 6, e39010615930, 2021

(CC BY 4.0) | ISSN 2525-3409 | DOI: http://dx.doi.org/10.33448/rsd-v10i6.15930

A comunicação aberta entre pais e filhos sobre a pandemia deve ser encorajada para ajudar adolescentes a lidar com problemas de saúde mental. Possivelmente, a percepção do impacto da quarentena domiciliar pode desempenhar um papel importante em relação à sintomas de ansiedade que, para adolescentes que estão confinados em suas casas devido ao fechamento de escolas durante a pandemia de COVID-19, tende a ser fator de risco (Tang, 2021). Estão sendo levadas em consideração sérias preocupações sobre a saúde mental, principalmente em relação ao aparecimento de sintomas de ansiedade em adolescentes durante a quarentena relacionada à pandemia de COVID-19, especialmente devido ao fechamento de escolas (Petzold, 2020).

Loades (2020) elucida que os serviços clínicos devem oferecer apoio preventivo e intervenção precoce sempre que possível, além de estarem preparados para o aumento dos problemas de saúde mental. Deve-se proporcionar uma individualização para cada paciente, levando-se em conta possíveis benefícios indiretos produzidos pela condição de restrição em crianças e adolescentes com algum tipo de transtorno prévio, tendo em vista o restabelecimento da rotina regular. É provável que esse suporte seja importante, reduzindo o impacto do distanciamento físico forçado, mantendo a estrutura, qualidade e quantidade das redes sociais e ajudando crianças e adolescentes a experimentar recompensas sociais, a se sentirem parte de um grupo e a saber que existem outros a quem eles podem procurar (Melegari, 2021).

Por fim, é importante orientar crianças e adolescentes a perceberem a pandemia por um ângulo construtivo e descobrir aspectos positivos em tempos difíceis pode ser uma forma adequada de ajudá-los a lidar com a pandemia (Chen, 2021).

\section{Estratégias inovadoras nas intervenções médicas em adolescente com ansiedade frente a pandemia por COVID-19}

Aplicar intervenções por meio de estratégias inovadoras na abordagem de adolescentes com sintomas de ansiedade frente à pandemia por COVID-19 torna-se um grande desafio para psiquiatras, pediatras e provedores de cuidados primários (Danese, 2020). Pensando nessa perspectiva, torna-se cada vez mais essencial efetuar o acompanhamento de adolescentes, família e cuidadores, com vistas a criar estratégias para mitigar os efeitos da pandemia do COVID-19.

Durante a pandemia por COVID-19 deve-se recomendar, sempre que possível, a exposição à natureza longe de aglomerações, pois os adolescentes que mantém exposição à natureza demonstram diminuição da ansiedade em comparação com aqueles que não tiveram muito acesso a estímulos externos semelhantes (Figueiredo, 2021).

Figueiredo (2021) traz em seus estudos que é preciso dar garantias de que adolescentes, assim como seus familiares, recebam o acompanhamento psiquiátrico e psicológico adequado para o suporte de sua saúde mental e, nessa tangente, a heterogeneidade e pluralidade de estressores psicossociais trazem a necessidade de uma abordagem multifatorial para a saúde mental de populações vulneráveis como adolescentes na pandemia por COVID-19.

Portanto, deve-se abordar o sofrimento emocional de adolescentes durante a pandemia e formular estratégias de promoção do bem-estar mental da população, com intervenções psicoeducativas universais para adolescentes e pais também com vários exemplos de materiais psicoeducativos online, preferencialmente aqueles com conselhos práticos, baseados em evidências e acessíveis (Duan, 2020). É importante envolver os adolescentes na coprodução dos materiais direcionados a eles e encontrar maneiras eficazes de engajar seus interesses e preferências em desenvolvimento, além de estabelecer políticas inovadoras de saúde mental para a faixa etária, com redes colaborativas diretas e digitais de profissionais e voluntários da comunidade (Singh, 2020).

É importante também destacar a eficácia do tratamento fornecido em telepsiquiatria contra os danos causados pela pandemia, para promover a saúde mental dos jovens. Além disso, os profissionais de saúde mental também podem desenvolver diretrizes para os pais sobre como ajudar seus filhos durante crises de saúde pública (Danese, 2020), a fim de reduzir o impacto negativo das consequências da pandemia na qualidade de vida dos adolescentes. 
Research, Society and Development, v. 10, n. 6, e39010615930, 2021

(CC BY 4.0) | ISSN 2525-3409 | DOI: http://dx.doi.org/10.33448/rsd-v10i6.15930

\section{Conclusão}

A pandemia de COVID-19 pode resultar em aumento de transtornos psiquiátricos, como estresse pós-traumático, transtornos depressivos e de ansiedade, bem como sintomas relacionados ao luto.

Nesta pesquisa não foram encontrados artigos contendo estudos que recomendassem intervenções com terapêutica medicamentosa primária. Em contrapartida, o diálogo entre familiares, amigos e colegas escolares, com o objetivo de aumentar a compreensão e aliviar o estresse, é recomendado para diminuir a ansiedade em adolescentes.

Percebe-se que o fortalecimento da divulgação do conhecimento da COVID-19 e das medidas de precaução adotadas para prevenir a disseminação da COVID-19 pode reduzir os níveis de ansiedade e depressão da população, principalmente nos adolescentes. As autoridades de saúde precisam identificar os grupos de alto risco para realizar intervenções psiquiátricas e psicológicas precoces.

Ainda há pouca literatura que tenha foco na ansiedade em adolescentes durante a pandemia. Faz-se necessário, portanto, que surjam novos estudos com foco nas estratégias de redução de ansiedade associada a este contexto mundial, a fim de que seja minimizado o impacto negativo na qualidade de vida dos adolescentes.

\section{Referências}

Ariapooran, S., \&Khezeli, M. (2021). Symptoms of anxiety disorders in Iranian adolescents with hearing loss during the COVID-19 pandemic. BMC psychiatry, 21(1), 114. https://doi.org/10.1186/s12888-021-03118-0

Castro A A. (2001). Revisão sistemática e meta-análise. Elaboração e Apresentação de Comunicação Científica. São Paulo.

Chen, X., Qi, H., Liu, R., Feng, Y., Li, W., Xiang, M., Cheung, T., Jackson, T., Wang, G., \&Xiang, Y. T. (2021). Depression, anxiety and associated factors among Chinese adolescentes during the COVID-19 outbreak: a comparisonoftwocross-sectionalstudies. Translational psychiatry, 11(1), 148.

Courtney, D., Watson, P., Battaglia, M., Mulsant, B. H., \&Szatmari, P. (2020). COVID-19 Impacts on Child and Youth Anxiety and Depression: Challenges and Opportunities. The Canadian Journal of Psychiatry, 65 (10), 688-691. https://doi.org/10.1177/0706743720935646

Danese, A., \& Smith, P. (2020). Debate: Recognisingandrespondingtothe mental healthneedsofyoungpeople in the era of COVID-19. Child and adolescent mental health, 25(3), 169-170. https://doi.org/10.1111/camh.12414

World Health Organization, WHO (2017). Depression and Other Common Mental Disorders: Global Health Estimates. Geneva: World Health Organization; Licence: CC BY-NC-SA 3.0 IGO. http://apps.who.int/bookorders

Duan, L., Shao, X., Wang, Y., Huang, Y., Miao, J., Yang, X., \& Zhu, G. (2020). Aninvestigation of mental health status of children and adolescents in china duringtheoutbreakof COVID-19. Journal of affective disorders, 275, 112-118. https://doi.org/10.1016/j.jad.2020.06.029

Figueiredo, C. S., Sandre, P. C., Portugal, L., Mázala-de-Oliveira, T., da Silva Chagas, L., Raony, Í., Ferreira, E. S., Giestal-de-Araujo, E., Dos Santos, A. A., \& Bomfim, P. O. (2021). COVID-19 pandemic impact on children andadolescents' mental health: Biological, environmental, and social factors. Progress in neuro-psycho pharmacology \& biological psychiatry, 106, 110171. https://doi.org/10.1016/j.pnpbp.2020.110171

Galvão, T. F., \& Pereira, M., G. (2014). Revisões sistemáticas da literatura: passos para sua elaboração. Epidemiologia e Serviços de Saúde, 23(1), $183-184$. http://scielo.iec.gov.br/scielo.php?script=sci_arttext\&pid=S1679-49742014000100018\&lng=pt\&tlng=pt.

Gambin, M. et. al. (2021). Generalized anxiety and depressive symptoms in various age groups duringthe COVID-19 lockdown in Poland. Specific predictors and differences in symptoms severity. Compr Psychiatry. 21(105), 152222. https://doi:10.1016/j.comppsych.2020.152222

Guessoum, S. B., Lachal, J., Radjack, R., Carretier, E., Minassian, S., Benoit, L., \& Moro, M. R. (2020). Adolescent psychiatric disorders during the COVID19 pandemic and lockdown. Psychiatry research, 291, 113264. https://doi.org/10.1016/j.psychres.2020.113264

Gunnell, D., Appleby, L., Arensman, E., Hawton, K., John, A., Kapur, N., Khan, M., O'Connor, R. C., Pirkis, J., \& COVID-19 Suicide Prevention Research Collaboration (2020). Suicide risk and prevention during the COVID-19 pandemic. The lancet. Psychiatry, 7(6), 468-471.

Loades, M. E., Chatburn, E., Higson-Sweeney, N., Reynolds, S., Shafran, R., Brigden, A., Linney, C., McManus, M. N., Borwick, C., \& Crawley, E. (2020). Rapid Systematic Review: The Impactof Social Isolation and Loneliness on the Mental Health of Children and Adolescents in the Context of COVID-19. Journal of the American Academy of Child and Adolescent Psychiatry, 59(11), 1218-1239.e3.

Melegari, M. G., Giallonardo, M., Sacco, R., Marcucci, L., Orecchio, S., \& Bruni, O. (2021). Identifying the impact of the confinement of Covid-19 on emotionalmood and behavioural dimensions in children and adolescents with attention deficit yperactivity disorder (ADHD). Psychiatry research, $296,113692$.

Petzold, M.B., et. al. (2020). Risk, resilience, psychological distress, and anxiety at the beginning of the COVID-19 pandemic in Germany. Brain and Behavior. 10(9), 01745. https://doi:10.1002/brb3.1745 
Research, Society and Development, v. 10, n. 6, e39010615930, 2021

(CC BY 4.0) | ISSN 2525-3409 | DOI: http://dx.doi.org/10.33448/rsd-v10i6.15930

Qi, H., Liu, R., Chen, X., Yuan, X. F., Li, Y. Q., Huang, H. H., Zheng, Y., \& Wang, G. (2020). Prevalence of anxiety and associated factors for Chinese adolescents during the COVID-19 outbreak. Psychiatry and clinical neurosciences, 74(10), 555-557.

Sher L. (2020). COVID-19, anxiety, sleep disturbances and suicide. Sleep medicine, 70, 124. https://doi.org/10.1016/j.sleep.2020.04.019

Singh, S. et. al. (2020). "Impact of COVID-19 and lockdown on mental health of children and adolescents: A narrative review wither commendations". Psychiatry research. 293 (2020), p. 113429.

Tang, S., Xiang, M., Cheung, T., \&Xiang, YT (2021). Saúde mental e seus correlatos entre crianças e adolescentes durante o fechamento da escola COVID-19: A importância da discussão entre pais e filhos. Journal of affective disorder, 279, 353-360. https://doi.org/10.1016/j.jad.2020.10.016

Thibaut F. (2017). Anxiety disorders: a review of current literature. Dialogues in clinical neuroscience, 19(2), 87-88. https://doi.org/10.31887/DCNS.2017.19.2/fthibaut.

Weisbrot, D. M., \&Ryst, E. (2020). Debate: Student mental health matters - the heightened need for school-based mental health in the era of COVID-19. Child and adolescent mental health, 25(4), 258-259. https://doi.org/10.1111/camh.12427

Zhou, S. J., Zhang, L. G., Wang, L. L., Guo, Z. C., Wang, J. Q., Chen, J. C., Liu, M., Chen, X., \& Chen, J. X. (2020). Prevalence and socio-demographic correlates of psychological health problems in Chinese adolescents during the outbreak of COVID-19. European child \& adolescent psychiatry, 29(6), 749-758. https://doi.org/10.1007/s00787-020-01541-4 